\title{
Knockdown of Slug by RNAi inhibits the proliferation and invasion of HCT116 colorectal cancer cells
}

\author{
JIANG QIAN, HONG LIU, WANGSHENG CHEN, KUNMING WEN, \\ WEIDONG LU, CHUN HUANG and ZHONGXUE FU \\ Department of General Surgery, The First Affiliated Hospital, Chongqing Medical University, \\ Chongqing 400016, P.R. China
}

Received December 12, 2012; Accepted April 9, 2013

DOI: $10.3892 / \mathrm{mmr} .2013 .1604$

\begin{abstract}
Colorectal cancer is one of the most common alimentary malignancies. Slug has been shown to be an ideal target for cancer gene therapy by numerous studies due to its strong anti-apoptotic effect. The elevated expression of Slug is a frequent genetic abnormality observed in colorectal cancer. In the present study, a Slug short hairpin RNA (shRNA) expression vector that was able to efficiently inhibit the expression of Slug in HCT116 cells was prepared. Following transfection, the mRNA expression levels were detected by RT-PCR analysis. Western blotting detected a similar inhibition of the Slug protein levels in the cells transfected with the pGCsi-Slug plasmid. Downregulation of Slug resulted in a significant inhibition of cancer cell growth in vitro. Cell invasion and apoptosis were decreased concomitantly with the reduction in Slug protein expression. These results suggested that RNA interference (RNAi) was able to downregulate the Slug protein level in HCT116 cells and significantly inhibit tumor growth in vitro. These findings suggest that RNAi has therapeutic potential for the treatment of colorectal cancer, as well as other types of cancer, by targeting the overexpression of oncogenes, including Slug.
\end{abstract}

\section{Introduction}

Colorectal cancer is one of the leading causes of cancer mortality worldwide (1). Colorectal cancer has become the second most common cancer in Asia (2). In Europe and the U.S., colorectal cancer accounts for $8-15 \%$ of all cancer mortality (3-5). Although traditional treatments for colorectal cancer, including surgical resection and chemotherapy, have greatly reduced the incidence of colorectal cancer in recent years, the survival rate remains unsatisfactory for the majority

Correspondence to: Dr Zhong-Xue Fu, Department of General Surgery, The First Affiliated Hospital, Chongqing Medical University, No. 1 Youyi Road, Yuanjiagang, Yuzhong, Chongqing 400016, P.R. China

E-mail: fzx990521@hotmail.com

Key words: Slug, RNA interference, colorectal cancer of patients with advanced cancer. In total, $\sim 50 \%$ of patient mortality is a result of the high recurrence and metastasis of tumors. One of the main reasons for the lack of effective therapies for these patients is our limited understanding of the exact molecular mechanisms involved in the carcinogenesis and progression of colorectal cancer. Therefore, finding novel therapeutic innovations against colorectal cancer is of great importance.

Slug is a member of the snail family of transcription factors and is expressed in the neural crest and in mesodermal cells emigrating from the primitive streak in chick embryos (6). It has previously been demonstrated that Slug is involved in the regulation of apoptosis and in the epithelial-mesenchymal transition (EMT), which is linked to the acquisition of an invasive phenotype $(7,8)$. Slug is also important in the regulation of EMT by the suppression of several epithelial markers and adhesion molecules, including E-cadherin. Studies have reported that Slug is expressed in breast carcinoma (9), cholangiocarcinoma $(10,11)$, bladder cancer (12), lung cancer (13) and oesophageal carcinoma $(14,15)$. These data demonstrate that the overexpression of Slug is correlated with the clinical aggressiveness of tumors and poor patient survival. Therefore, researchers have considered that Slug may be a significant prognostic biomarker for colorectal cancer. A family of proteolytic enzymes that degrade the extracellular matrix (ECM) or components of the basement membrane have been associated with tumor cell invasion, angiogenesis and metastasis in bladder cancer (16). Slug levels have been correlated with tumor progression and invasion in human colorectal cancer, as well as with poor patient survival. Slug expression in colorectal cancer has also been observed to be an independent prognostic factor for metastasis and survival (17). Thus, Slug is considered as an ideal target for colorectal cancer gene therapy. In the present study, RNA interference (RNAi) was used to investigate the effect of Slug on the function of the human colorectal cancer HCT116 cell line. The proliferation, apoptosis and invasion of the HCT116 cells in vitro were examined.

\section{Materials and methods}

Cell lines. The human colorectal cancer HCT116 cell line was obtained from the Shanghai Institute of Cell Biology, 
Chinese Academy of Sciences (Shanghai, China). The cells were cultured in RPMI-1640 (Gibco-BRL, Paisley, UK) and the antibiotics were supplemented with $10 \%$ fetal bovine serum (FBS; Hyclone, Logan, UT, USA) and $100 \mathrm{U} / \mathrm{ml}$ each of penicillin and streptomycin. All cells were maintained in a humidified incubator at $37^{\circ} \mathrm{C}$ with $5 \% \mathrm{CO}_{2}$ and stored at $-20^{\circ} \mathrm{C}$. The cells were fed every 2-3 days with complete medium and subcultured when confluence was reached.

Short hairpin RNA (shRNA) preparation and vector-based shRNA plasmid construction. The Slug-specific small interfering RNA (siRNA) was designed to target the sequence 5'-CCTCACTGCAACAG AGCATTT-3' of the Slug transcript (GenBank Accession no. NM_003068) and was synthesized by GeneChem (Shanghai, China). The siRNA sequence was determined to be unique to the human gene by Basic Local Alignment Search Tool (BLAST) searches of the GenBank database and did not show any homology to any other sequence. The series (no. NM_003068) and a single non-silencing luciferase sequence were combined into a shRNA oligonucleotide template consisting of sense, hairpin loop, antisense and terminator sequences, all of which were flanked by restriction enzyme sites to facilitate directional subcloning. The shRNAs were subcloned into the plasmid pGCsi shRNA expression vector by GeneChem. The resulting vectors were encoded for green fluorescent protein (GFP) under the transcriptional control of the cytomegalovirus (CMV) promoter and either shRNA directed against Slug or a non-silencing luciferase shRNA under the U6 promoter.

Plasmid transfection in vitro. The HCT116 cells were divided into three groups: Cells transfected with PBS as the control group (the CON group, noninfection), cells transfected with pGCsi as the negative control group (the NC group) and cells transfected with pGCsi-Slug as the experimental group (the KD group). The HCT116 cells were trypsinized and cultured in 24 -well plates $\left(2 \times 10^{5}\right.$ cells/well). At $24 \mathrm{~h}$ following seeding, the cells were seeded in RPMI-1640 medium without antibiotics until they reached $40-60 \%$ confluence; they were then transfected with pGCsi-Slug using Lipofectamine 2000 (Invitrogen Life Technologies, Carlsbad, CA, USA), according to the manufacturer's instructions. Briefly, $15 \mu \mathrm{l}$ pGCsi-Slug $(20 \mu \mathrm{M})$ and $5 \mu \mathrm{l}$ Lipofectamine 2000 were diluted, individually, in $250 \mu \mathrm{l}$ of Opti-MEM. Subsequent to a 5-min incubation at room temperature, the diluted pGCsi-Slug and the Lipofectamine were gently combined and incubated for $20 \mathrm{~min}$ at room temperature to allow complex formation. The pGCsi-Slug Lipofectamine complexes were then added to the plated cells and mixed gently. Lipofectamine and the Plus transfection reagent (Invitrogen Life Technologies) were used for transfection in the blank control group. The cells were incubated at $37^{\circ} \mathrm{C}$ in a humidified atmosphere of $5 \% \mathrm{CO}_{2}$ for $24 \mathrm{~h}$ and then the transfection efficiency was observed using fluorescence microscopy.

RT-PCR analysis. RT-PCR was used for the analysis of the Slug mRNA with GAPDH as an internal control. The total RNA was isolated from the HCT116 cells using TRIzol (Invitrogen Life Technologies) at $48 \mathrm{~h}$ post-transfection. The primers used for Slug were as follows: forward primer, 5'-CAAGGACACATTAGAACTCACAC-3' and reverse primer, 5'-CTACACAGCAGCCAGATTC-3'. The GAPDH primers were: forward primer, 5'-TGACTT CAACAGCGACACCCA-3' and reverse primer, 5'-CACCCT GTTGCTGTAGCCAAA-3'. The reactions were performed in accordance with the standard PCR protocol. One-step RT-PCR (Takara Bio Inc., Shiga, Japan) was performed at $50^{\circ} \mathrm{C}$ for $30 \mathrm{~min}$ and $2 \mathrm{~min}$ at $95^{\circ} \mathrm{C}$, followed by 40 cycles of $30 \mathrm{sec}$ at $95^{\circ} \mathrm{C}, 30 \mathrm{sec}$ at $60^{\circ} \mathrm{C}$ and $45 \mathrm{sec}$ at $72^{\circ} \mathrm{C}$. The products were separated by electrophoresis in $3 \%$ agarose and visualized with ethidium bromide. The intensity of the bands was analyzed using Quantity One software (Bio-Rad, Hercules, CA, USA). All of the experiments were repeated at least three times.

Western blot analysis. The cells from each group were washed twice with PBS following transfection for $48 \mathrm{~h}$ and then harvested with a lysis buffer containing phosphatase and protease inhibitors. The cell protein extracts were separated by SDS-PAGE and transferred onto nitrocellulose membranes, using standard techniques. The immobilized proteins were blocked in sterilized $0.5 \%$ gelatin in Tris-buffered saline $/ 0.1 \%$ Tween-20 (TBST) and then the primary polyclonal antibodies against Slug (Cell Signaling Technology, Inc., Beverly, MA, USA) were incubated at $4^{\circ} \mathrm{C}$ overnight. The membranes were incubated with a secondary antibody (goat anti-rabbit) according to the manufacturer's instructions. Subsequent to iterative washing with TBST, the complexes were visualized by the Odyssey Infrared Imaging System (LICOR, Lincoln, NE, USA). All of the experiments were repeated on at least three occasions.

Cell proliferation assay. To evaluate the effect of the siRNAs on the proliferation of the HCT116 cells, following 3 days of infection the cells were trypsinized, resuspended and the transiently transfected cells $\left(\sim 5 \times 10^{4}\right.$ cells $)$ were seeded into each well of the 96 -well plates and cultured for $24 \mathrm{~h}$. Following this, 3-(4,-dimethylthiazol-2-yl)-2,5-diphenyltetrazolium bromide (MTT; DingGuo Biotech, Beijing, China) was added to $100 \mu \mathrm{l}$ $(0.5 \mathrm{mg} / \mathrm{ml}$ in PBS) of medium in each well after $48 \mathrm{~h}$. The plates were incubated for $4 \mathrm{~h}$ at $37^{\circ} \mathrm{C}$ until purple formazan crystals developed. Subsequently, the MTT-containing medium was discarded and $150 \mu \mathrm{l}$ DMSO solution was added to each well to dissolve the formazan crystals. The plates were then incubated at room temperature for $30 \mathrm{~min}$. The absorbance was measured at $490 \mathrm{~nm}$ using a spectrophotometer (Thermo, Waltham, MA, USA). The experiments were performed in triplicate. The results are shown as the mean \pm SD of the three independent experiments.

Cell invasion assay. The cell invasive capability in vitro was evaluated in a transwell chamber assay. A total of $60 \mu 1$ diluted ECM gel solution was placed into the upper chambers of the transwell inserts (24-well insert, $8 \mu \mathrm{m}$ pore size; BD Biosciences, Bedford, MA, USA). The inserts were incubated at $37^{\circ} \mathrm{C}$ for $4 \mathrm{~h}$ for gelling and then pretreated with serum-free DMEM at $37^{\circ} \mathrm{C}$ for $1 \mathrm{~h}$ prior to seeding the cells at a density of $5 \times 10^{6} /$ well in $100 \mu \mathrm{l}$ culture medium. A total of $500 \mu \mathrm{l}$ DMEM containing $10 \%$ fetal calf serum was added to the lower chamber. Following $20 \mathrm{~h}$ of incubation, the non-invaded 
cells were removed from the inner section of the insert with a cotton swab. The cells at the bottom of the ECM gel-coated membrane were fixed and stained with paraformaldehyde and hexamethyl pararosaniline chloride. The hexamethyl pararosaniline chloride was dissolved in $200 \mu \mathrm{l}$ glacial acetic acid and incubated at room temperature for $30 \mathrm{~min}$. The cells that invaded through the membrane were counted using fluorescence microscopy (magnification, $\mathrm{x} 100$ ) by randomly selecting five fields per membrane. The invasive activity of the cells was expressed as the mean number of cells that invaded the lower sides of the chambers. The experiments were performed three times.

Colony formation assay. The cells in all three groups were trypsinized and resuspended. A concentration of 200 cells per well were then mixed with a $0.3 \%$ agar solution in DMEM containing $10 \% \mathrm{FBS}$ and layered on top of a $0.6 \%$ agar layer seeded into a 6 -well plate. The plates were then incubated for 14 days at $37^{\circ} \mathrm{C}$ in $5 \% \mathrm{CO}_{2}$ to form colonies. The media were changed every 3-4 days. At the end of incubation, the cells were washed twice with PBS and fixed with paraformaldehyde. The cells were then washed again twice with PBS, stained with Giemsa (Sigma, St. Louis, MO, USA) for $10 \mathrm{~min}$ and washed with $\mathrm{ddH}_{2} \mathrm{O}$ three times, sequentially. Images of the plates were captured with a digital camera. The results are shown as the mean number of colonies observed in five wells. The experiments were all performed three times.

Statistical analysis. The results of each experiment are represented as the mean \pm standard deviation (SD) of the three independent experiments. The statistical analysis was performed using the SPSS statistical software (SPSS, Inc., Chicago, IL, USA). A Student's two-tailed t-test was used to determine any differences in the means between the two groups. $\mathrm{P}<0.05$ was considered to indicate a statistically significant difference.

\section{Results}

pGCsi-Slug transfection efficiency. To investigate the lentiviral infection efficiency, the pGCsi-Slug expression vector was transfected into the HCT116 cells. At 48 h post-transfection, the protein expression of GFP was detected with fluorescence microscopy. The resulting fluorescence expression was identified in $>90 \%$ of the HCT116 cells following infection (Fig. 1). This result demonstrated the high efficiency of pGCsi-Slug.

pGCsi-Slug expression vector inhibits the expression of Slug at the mRNA and protein levels in vitro. The transient knockdown efficiencies of pGCsi-Slug in the HCT116 cells were detected by RT-PCR and western blotting (Fig. 2). At $48 \mathrm{~h}$ post-transfection, the relative Slug mRNA level was normalized against the mRNA level of an internal control gene that was performed in the same run (duplex assay). As shown in Fig. 2A, a marked inhibition of the Slug mRNA expression was observed in the KD group compared with the $\mathrm{NC}$ and CON groups. The relative levels of Slug mRNA in the CON, NC and KD groups were $1.012 \pm 0.05,0.937 \pm 0.06$ and $0.271 \pm 0.02$, respectively. Statistical analysis showed that the expression of the Slug mRNA in the KD group was significantly downregulated subsequent to transfection with pGCsi-Slug $(\mathrm{P}<0.01)$, whereas the two other groups $(\mathrm{CON}$ and NC) demonstrated no effect on the expression of Slug mRNA ( $P>0.05)$. With regard to the Slug protein expression levels, a marked inhibition of Slug protein expression was observed in the KD group compared with the other two groups. At $48 \mathrm{~h}$ post-transfection, the relative levels of the Slug protein in the cells of the CON, NC and KD groups were $0.56 \pm 0.05$, $0.53 \pm 0.046$ and $0.12 \pm 0.03$, respectively $(\mathrm{P}<0.01$; Fig. $2 \mathrm{~B}$ and 2C). These results showed that a Slug-silenced HCT116 stable cell line was generated in which Slug expression at the mRNA and protein levels was suppressed.

Knockdown of Slug inhibits the proliferation and colony formation of HCT116 cells. To examine the effect of pGCsi-Slug on the cell proliferation of HCT116 cells post-transfection, the cell growth curves obtained by the MTT assay were plotted. As shown in Fig. 3A, the growth rate was significantly lower in the KD group compared with the CON and NC groups at 4, 5, 6 and 7 days. There was no significant difference between the $\mathrm{CON}$ and $\mathrm{NC}$ groups. The colony formation rate of the pGCsi-Slug-transfected cells $(20 \pm 2.35$ cells/field $)$ was also markedly suppressed compared with the CON $(58 \pm 3.58$ cells/field) and NC (56 \pm 3.64 cells/field) groups. Additionally, the clones in the KD group were notably smaller compared with those in the control cells (Fig. 3B). These results demonstrated that pGCsi-Slug suppressed HCT116 cell proliferation in the cell culture.

Knockdown of Slug inhibits the invasion of HCT116 cells. An invasion assay was conducted using the transwell chamber assay, which was based on findings that showed that the movement of cells through matrigel-coated membrane mimicked the early step of tumor invasion. As shown in Fig. 4, subsequent to 2 days of incubation, the number of cells that invaded through the artificial basement membrane was $85.69 \pm 4.32$ cells/field in the $\mathrm{NC}$ group, $80.23 \pm 3.86$ cells/field in the CON group and $25.31 \pm 2.31$ cells/field in the KD group. The results indicated that the number of cells invading through the transwell membrane was significantly lower in the KD group compared with that in the NC and CON groups $(\mathrm{P}<0.01)$. There was no significant difference between the $\mathrm{NC}$ and $\mathrm{CON}$ groups $(\mathrm{P}>0.05)$. Thus, downregulation of Slug gene expression resulted in a marked inhibition of the invasion capacity of the HCT116 cells.

\section{Discussion}

Cancer cells often demonstrate an alteration in certain signal transduction pathways, leading to proliferation in response to external signals. Oncogene overexpression is a common phenomenon in the development and progression of several types of cancers. Therefore, oncogenes provide a potential target for cancer gene therapy (18). Slug is involved in cell division and invasion, and the overexpression of Slug correlates with the poor prognosis of colon cancer patients. Slug is a member of the snail family of zinc-finger transcriptional factors and is expressed in the neural crest and in mesodermal cells emigrating from the primitive streak in 


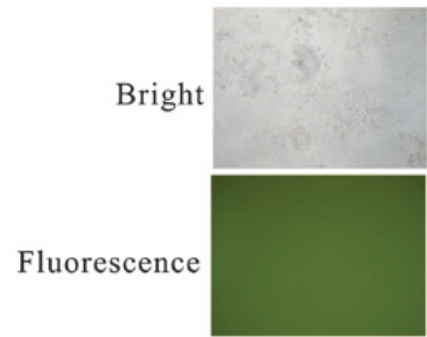

CON

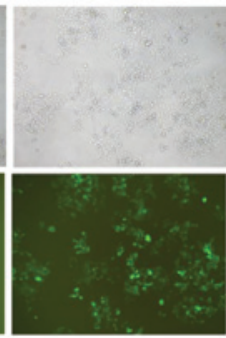

NC

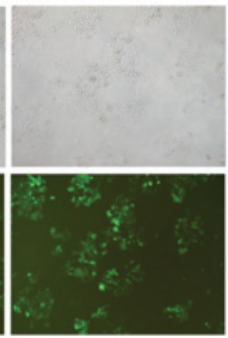

$\mathrm{KD}$
Figure 1. Detection of lentiviral infection efficiency. The HCT116 cells expressing GFP at $48 \mathrm{~h}$ post-transfection. GFP, green fluorescent protein; $\mathrm{CON}$, blank control; NC, nonsense short hairpin RNA; KD, pGCsi-Slug plasmid.

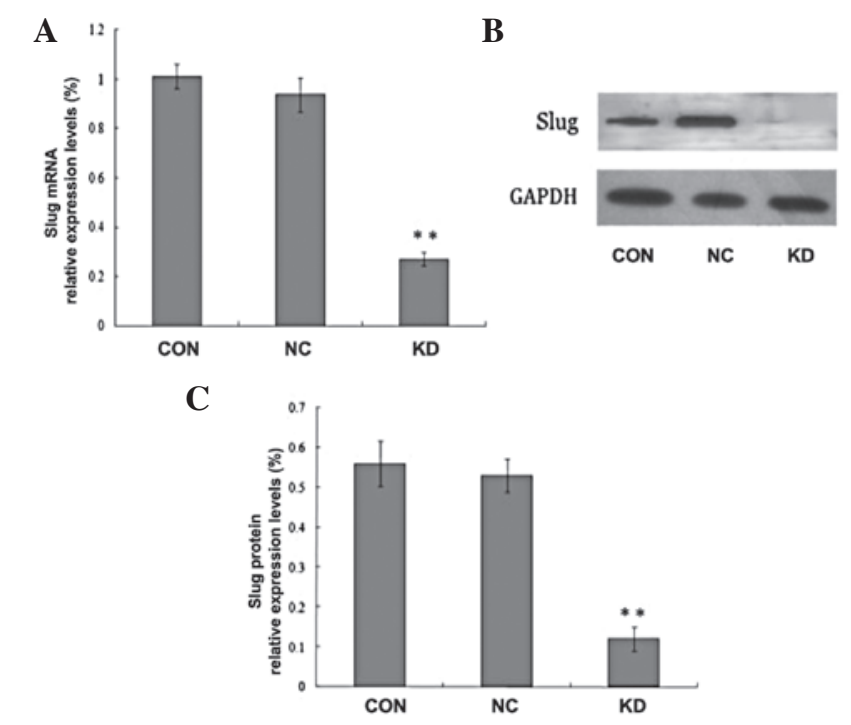

Figure 2. pGCsi-Slug plasmid stably inhibited Slug mRNA and protein expression in the HCT116 cells. (A) Slug mRNA was detected by RT-PCR following RNAi treatment. GAPDH was used as an internal control. (B) Slug protein was detected by western blotting following RNAi treatment. GAPDH was used as an internal control. (C) Bar graph of the western blotting results. ${ }^{* *} \mathrm{P}<0.01$, compared with the CON and NC groups. RNAi, RNA interference; CON, blank control; NC, nonsense short hairpin RNA; KD, pGCsi-Slug plasmid.

chick embryos (7). Slug is highly expressed in cells undergoing EMT in the developing chick embryo, where it plays a critical role in the formation of the primitive streak, endocardial cushions, decondensing somites and the closure of the palate. Experimental data have led to the inclusion of Slug into the Snail family of transcription regulators involved in tumor progression and metastasis $(15,19)$. A previous study suggested that patients with positive Slug expression had a poor clinical outcome for esophageal squamous cell carcinoma (15). In addition, the clinical association of Slug with colorectal cancer has been studied (17). These data showed that Slug was overexpressed in human colorectal cancer and may be involved in the processes of cancer invasion and metastasis. However, the essential roles of Slug in colorectal cancer remain unclear.

In the present study, to clarify the role of Slug in colorectal cancer, Slug expression was suppressed by RNAi in HCT116 cells. Cell growth and plate colony formation assays were applied to assess the cell proliferative potential following the inhibition of Slug expression. The results showed that
$\mathbf{A}$

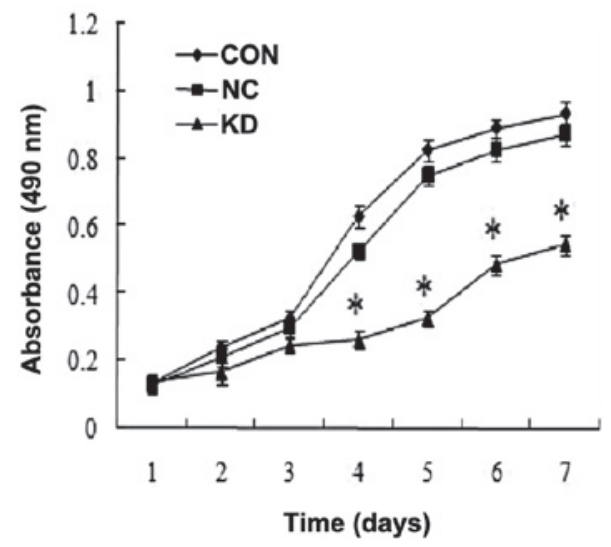

B
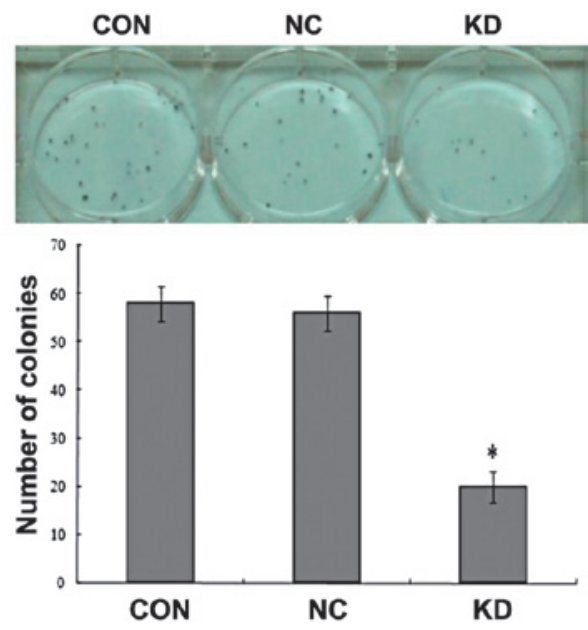

Figure 3. Effect of Slug RNAi on cell growth. (A) Absorbance and (B) colony formation of the HCT116 cells. ${ }^{*} \mathrm{P}<0.05$, compared with the CON and NC groups. CON, blank control; NC, nonsense short hairpin RNA; RNAi, RNA interference; $\mathrm{KD}$, pGCsi-Slug plasmid.

A CON NC KD
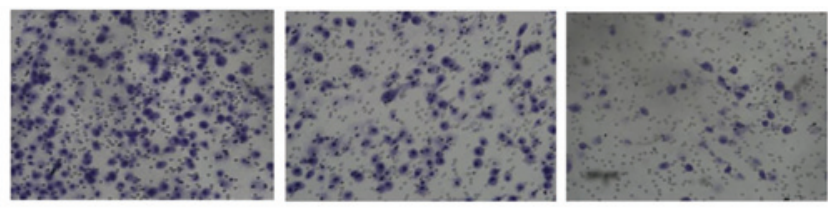

B

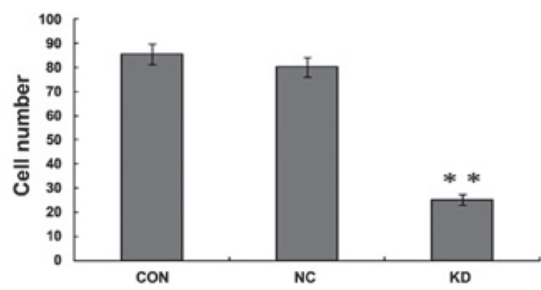

Figure 4. Effect of silencing the Slug gene on in vitro cell invasion ability. (A) Cells were stained and counted under a microscope by randomly selecting five fields per membrane. All experiments were performed in triplicate. (B) Bar graph showing the mean values of the three independent experiments. ** $\mathrm{P}<0.01, \mathrm{KD}$ group compared with the $\mathrm{NC}$ and $\mathrm{CON}$ groups. CON, blank control; NC, nonsense short hairpin RNA; KD, pGCsi-Slug plasmid.

the downregulation of Slug expression significantly inhibited the proliferation of HCT116 cells in vitro. Previously, an abundance of studies have shown that EMT is important in tumor progression and metastasis. According to the results 
of previous studies, Slug is able to modulate the expression of several genes involved in EMT and is an essential regulator during the invasion and metastasis of tumors (14). The previous study also assessed the invasive abilities of untransfected and transfected HCT116 cells. The results indicated that the downregulation of Slug expression was able to significantly decrease the invasive ability of HCT116 cells. These data suggest that the Slug gene may be a significant factor in colorectal cancer growth and invasion, since changes in cell proliferation and apoptosis are key factors in regulating neoplastic progression. These results are similar to those reported in previous studies examining other types of cancer (16) and suggest that Slug may have a semblable effect on the growth and invasion of several types of human malignant epithelial tumors.

In conclusion, the present study has demonstrated that the Slug gene is able to regulate the proliferation and invasion of human colorectal cancer HCT116 cells in vitro. Although additional in vitro and in vivo studies are required, these data suggest that the reduction of Slug expression may provide a potential therapeutic approach to regulate the proliferation and invasion of colorectal cancer in patients with Slug overexpression. Although metastasis formation in colon cancer, as well as in other types of cancer, is a highly complex and organized process that consists of multiple inter-related steps, Slug appears to play a significant role.

\section{References}

1. Jemal A, Bray F, Center MM, Ferlay J, Ward E and Forman D: Global cancer statistics. CA Cancer J Clin 61: 69-90, 2011.

2. Sung JJ, Choi SY, Chan FK, Ching JY, Lau JT and Griffiths S: Obstacles to colorectal cancer screening in Chinese: a study based on the health belief model. Am J Gastroenterol 103: 974-981, 2008

3. Cohen SJ, Cohen RB and Meropol NJ: Targeting signal transduction pathways in colorectal cancer - more than skin deep. J Clin Oncol 23: 5374-5385, 2005

4. Waters C: Colon cancer: an overview. Pharmaceut J 276: 323-326, 2006.
5. Labianca R, Beretta G, Gatta G, de Braud F and Wils J: Colon cancer. Crit Rev Oncol Hematol 51: 145-170, 2004.

6. Nieto MA: The snail superfamily of zinc-finger transcription factors. Nature Rev Mol Cell Biol 3: 155-166, 2002.

7. Nieto MA, Sargent MG, Wilkinson DG and Cooke J: Control of cell behavior during vertebrate development by Slug, a zinc finger gene. Science 264: 835-839, 1994.

8. Haupt S, Alsheich-Bartok O and Haupt Y: Clues from worms: a Slug at Puma promotes the survival of blood progenitors. Cell Death Differ 13: 913-915, 2006.

9. Martin TA, Goyal A, Watkins G and Jiang WG: Expression of the transcription factors snail, slug, and twist and their clinical significance in human breast cancer. Ann Surg Oncol 12: 488-496, 2005.

10. Zhang KJ, Wang DS, Zhang SY, Jiao XL, Li CW, Wang XS, $\mathrm{Yu}$ QC and Cui HN: The E-cadherin repressor slug and progression of human hilar cholangiocarcinoma. J Exp Clin Cancer Res 29: 88, 2010.

11. Zhang KJ, Zhang BY, Zhang KP, Tang LM, Liu SS, Zhu DM and Zhang DL: Clinicopathologic significance of slug expression in human intrahepatic cholangiocarcinoma. World J Gastroenterol 16: 2554-2557, 2010.

12. Yu Q, Zhang K, Wang X, Liu X and Zhang Z: Expression of transcription factors snail, slug, and twist in human bladder carcinoma. J Exp Clin Cancer Res 29: 119, 2010.

13. Shih YJ, Tsai MF, Chang TH, Chang YL, Yuan A, Yu CJ, Lin SB, Liou GY, Lee ML, Chen JJ, Hong TM, Yang SC, Su JL, Lee YC, and Yang PC: Transcription repressor slug promotes carcinoma invasion and predicts outcome of patients with lung adenocarcinoma. Clin Cancer Res 11: 8070-8078, 2005.

14. Zhang K, Zhang S, Jiao X, Wang H, Zhang D, Niu Z, Shen Y, Lv L and Zhou Y: Slug regulates proliferation and invasiveness of esophageal adenocarcinoma cells in vitro and in vivo. Med Oncol 28: 1089-1100, 2011.

15. Uchikado $Y$, Natsugoe S, Okumura H, Setoyama $T$, Matsumoto M, Ishigami S and Aikou T: Slug expression in the E-cadherin preserved tumors is related to prognosis in patients with esophageal squamous cell carcinoma. Clin Cancer Res 11: 1174-1180, 2005.

16. Kanayama H: Matrix metalloproteinases and bladder cancer. J Med Invest 48: 31-43, 2001.

17. Shioiri M, Shida T, Koda K, Oda K, et al: Slug expression is an independent prognostic parameter for poor survival in colorectal carcinoma patients. Br J Cancer 94: 1816-1822, 2006.

18. Chung-Faye GA and Kerr DJ: Innovative treatment for colon cancer. BMJ 321: 1397-1399, 2000.

19. Peinado H, Olmeda D and Cano A: Snail, Zeb and bHLH factors in tumour progression: an alliance against the epithelial phenotype? Nat Rev Cancer 7: 415-428, 2007. 